Journal of Universal Mathematics

Vol.3 No.1 Pp.66-76 (2020)

ISSN-2618-5660

\title{
ON SOME NEW $F K$ SPACES OBTAINED FROM SUMMABILITY MATRIX
}

\author{
MAHMUT KARAKUŞ AND TUNAY BİLGIN
}

\begin{abstract}
In this study, we give some new $F K$-spaces by means of an infinite matrix as an operator and define some new $\beta$ - and $\gamma$-type duality of sequence spaces $[4,6]$. We also introduce some new sections and investigate some properties like $A B-, F A K-, S A K$ - and $A K$ - in an $F K$-space. By this way, we obtain some new distinguished subspaces of an $F K$-space [7]. Among other results, we prove that the sum of finite numbers of $F K$-spaces and the intersection of a sequence of $F K$-spaces which have these new properties with corresponding paranorms have also these new properties. The reader can refer to [2] and [19] for the main results and related topics in FK-space theory.
\end{abstract}

\section{Preliminaries and Notation}

The space of all scalar valued sequences is given by $\omega$ and a $K$ space is a locally convex sequence space (lcss) $\lambda$ containing $\phi$ and a subspace of $\omega$ on which coordinate functionals $\pi_{k}(x)=x_{k}$ are continuous for every $k \in \mathbb{N}$. Here $\phi$ is the space of finitely non-zero sequences spanned by $\left\{\left(\delta^{k}\right): k \in \mathbb{N}\right\}$ which is the space of sequences whose $k$ th position is 1 and all the others are 0 . A complete linear metric (or complete normed linear) $K$ space is called an $F K$ (or $B K$ ) space.

The multipliers from $\lambda$ into $\mu$ are given by $\lambda^{\mu}=\{y \in \omega \mid x y \in \mu, \forall x \in \lambda\}$ for $\lambda, \mu \subset \omega$, where $x y$ is the coordinatewise product, i.e., $x y=\left\{x_{k} y_{k}\right\}_{k \in \mathbb{N}^{*}}$. We notates $\left(\lambda^{\mu}\right)^{\nu}=\lambda^{\mu \nu}=\left\{y \in \omega \mid x y \in \nu, \forall x \in \lambda^{\mu}\right\}$ for $\lambda, \mu, \nu \subset \omega$. A sequence space $\lambda$ is called $\mu$-perfect if $\lambda=\lambda^{\mu \mu}$. Classical $\alpha-, \beta$ - and $\gamma$ - duals of $\lambda$ are given by $\lambda^{\ell}, \lambda^{c s}$ and $\lambda^{b s}$, respectively, where $\ell=\left\{\left(x_{k}\right) \in \omega:\|x\|_{1}=\sum_{k}\left|x_{k}\right|<\infty\right\}$, cs $=\left\{\left(x_{k}\right) \in\right.$ $\omega: \sum_{k} x_{k}$ is convergent $\}$ and $b s=\left\{\left(x_{k}\right) \in \omega:|| x \|_{b s}=\sup _{n}\left|\sum_{k=1}^{n} x_{k}\right|<\infty\right\}$. These are Banach spaces with their natural norms and also $c s$ is Banach spaces with $\|.\|_{b s}$. We know, $\phi \subset \lambda^{\alpha} \subset \lambda^{\beta} \subset \lambda^{\gamma}$. If $\lambda \subset \mu$ then $\mu^{\zeta} \subset \lambda^{\zeta}$ and for every $\lambda$ we have $\lambda^{\zeta}=\lambda^{\zeta \zeta \zeta}, \lambda \subset \lambda^{\zeta \zeta}$, where $\zeta$ is one of the $\alpha-, \beta-$ or $\gamma-\mathrm{du}-$ als. Let us note, Fleming and Magee showed that, whenever $\lambda \supset \phi$ is a sequence space (not required be a $B K$ ) and $\mu \supset \phi$ is a $B K$ space then $\lambda^{\mu}$ is a $B K$ space iff there exist a norm $\|$.$\| on \lambda$ such that for every $y \in \lambda^{\mu}$ the diagonal map $T_{y}: \lambda \rightarrow \mu, T_{y}(x)=x y$ is continuous with respect to this norm [9]. We denote $f$-dual of a $B K$ space $\lambda \supset \phi$ with $\lambda^{f}=\left\{\left(f\left(\delta^{k}\right)\right)_{k \in \mathbb{N}} \mid \exists f \in \lambda^{\prime}\right\}$. Here $\lambda^{f}$ is also a $B K$ space with $\|f\|_{\lambda^{\prime}}=\left\|\left(f\left(\delta^{k}\right)\right)_{k \in \mathbb{N}}\right\|_{\lambda^{f}}$. A $K$ space $\lambda \supset \phi$ is called a sum space

2000 Mathematics Subject Classification. 40H05; 46A45; 40G99; $40 \mathrm{C} 05$.

Key words and phrases. FK spaces, Matrix methods, $\beta-, \gamma-, f$-duality. 
if $\lambda^{\lambda}=\lambda^{f}$. For example, $\ell, c s$ and $b s$ are $B K$ sum spaces. If $\lambda \supset \phi$ is a $K$ space then $S \in \lambda^{\prime}$ is called a sum on $\lambda$ if $S\left(\delta^{k}\right)=1, \forall k \in \mathbb{N}$ or equivalently $S$ is a sum on $\lambda$ if $S(x)=\sum x, \forall x \in \phi$, where $S \in \lambda^{\prime}$. A $K$ space $\lambda$ is called $A D$ space if $\lambda=\bar{\phi}$, where $\bar{\phi}$ is closure of $\phi$ in $\lambda$. Via Hahn-Banach theorem, $\lambda^{f}=\bar{\phi}^{f}$.

Let $\lambda=\left(\lambda_{k}\right)$ be a strictly increasing sequence of positive real numbers tending to infinity, that is,

$$
0<\lambda_{1}<\lambda_{2}<\cdots \text { and } \lim _{k \rightarrow \infty} \lambda_{k}=\infty .
$$

Then the sequence $x=\left(x_{k}\right) \in \omega$ is said to be $\lambda$-convergent to the number $a \in \mathbb{C}$, if $(\Lambda x)_{n} \rightarrow a$, as $n \rightarrow \infty$; where

$$
(\Lambda x)_{n}=\frac{1}{\lambda_{n}} \sum_{k=1}^{n}(\Delta \lambda)_{k} x_{k}
$$

for all $n \in \mathbb{N}$. Throughout the text we shall assume that $(\Delta \lambda)_{k}=\lambda_{k}-\lambda_{k-1}$ for all $k \in \mathbb{N}$ and $\lambda_{0}=0$. The set $c^{\lambda}$ of all $\lambda$ convergent sequences is a $B K$ space with the norm $\|x\|_{\ell_{\infty}^{\lambda}}=\|\Lambda x\|_{\infty}=\sup _{n \in \mathbb{N}}\left|(\Lambda x)_{n}\right|$, where $\Lambda x=\left\{(\Lambda x)_{n}\right\}$; [15]. The matrix $\Lambda=\left(\lambda_{n k}\right)$ is also defined by

$$
\lambda_{n k}:=\left\{\begin{array}{cll}
\frac{\lambda_{k}-\lambda_{k-1}}{\lambda_{n}} & , \quad(1 \leq k \leq n), \\
0 & , \quad(k>n)
\end{array}\right.
$$

for all $k, n \in \mathbb{N}$. In the special case $\lambda_{n}=n$ for all $n \in \mathbb{N}$, the $\Lambda$-matrix is reduced to the Cesàro matrix $C$ of order one. We also note that $\Lambda$-summability is the special case of the $\bar{N} q$-summability; [15], (see also [2]).

Lemma 1.1. $c_{0} \subset c_{0}^{\lambda}, c \subset c^{\lambda}$ and $\ell_{\infty} \subset \ell_{\infty}^{\lambda}$ strictly hold if and only if

$$
\liminf _{n} \frac{\lambda_{n+1}}{\lambda_{n}}=1
$$

[15].

Lemma 1.2. $c_{0}=c_{0}^{\lambda}, c=c^{\lambda}$ and $\ell_{\infty}=\ell_{\infty}^{\lambda}$ hold if and only if

$$
\liminf _{n} \frac{\lambda_{n+1}}{\lambda_{n}}>1
$$

[15].

\section{Some New Sections and Distinguished Subspaces of an $F K$-spaces}

Let $x=\left(x_{k}\right) \in \omega$ be a sequence, then by using $\Lambda=\left(\lambda_{n k}\right)$, we have $\Lambda n^{t h}$ section of $x$ as;

$$
x_{\lambda}^{[n]}=\Lambda\left(x^{[k]}\right)=\frac{1}{\lambda_{n}} \sum_{k=1}^{n}\left(\Delta \lambda_{k}\right) x^{[k]} .
$$

Here, $x^{[k]}=\sum_{j=1}^{k} x_{j} \delta^{j}$ and $\Delta \lambda_{k}=\lambda_{k}-\lambda_{k-1},\left(k \in \mathbf{N}\right.$ and $\left.\lambda_{0}=0\right)$.

A sequence $x$ in any $K$ space $X \supset \phi$ has $\lambda A K$ property if $x_{\lambda}^{[n]} \rightarrow x,(n \rightarrow \infty)$ in $X$ and we say $X$ is an $\lambda A K$ - space if all elements of $\lambda$ have this property. Similarly 
we can define the properties, $S \lambda A K, F \lambda A K$ and $\lambda A B$. So, we define the following sets as:

$$
\begin{aligned}
\Lambda S_{X} & =\left\{x \in X \mid x=\lim _{n} x_{\lambda}^{[n]}\right\}, \\
\Lambda W_{X} & =\left\{x \in X \mid x_{\lambda}^{[n]}-x \text { in } \lambda\right\}("-\text { " means weakly }) \\
& =\left\{x \in X \mid f(x)=\lim _{n} f\left(x_{\lambda}^{[n]}\right), \forall f \in X^{\prime}\right\}, \\
\Lambda F_{X}^{+} & =\left\{x \in \omega \mid\left(x_{\lambda}^{[n]}\right)_{n \in \mathbb{N}} \text { weakly Cauchy in } X\right\} \\
& =\left\{x \in \omega \mid\left(f\left(x_{\lambda}^{[n]}\right)\right)_{n \in \mathbb{N}} \in c, \forall f \in \lambda^{\prime}\right\}, \\
\Lambda B_{X}^{+} & =\left\{x \in \omega \mid\left(x_{\lambda}^{[n]}\right)_{n \in \mathbb{N}} \text { is bounded in } X\right\} \\
& =\left\{x \in \omega \mid\left(f\left(x_{\lambda}^{[n]}\right)\right)_{n \in \mathbb{N}} \in \ell_{\infty}, \forall f \in X^{\prime}\right\} .
\end{aligned}
$$

One should keep in mind that $\Lambda B_{X}=\Lambda B_{X}^{+} \cap X$ and $\Lambda F_{X}=\Lambda F_{X}^{+} \cap \lambda$. These are the spaces of the sequences which have $\lambda A B$ and $F \lambda A K$, respectively. Now for example, if the normed sequence space $X$ is an $\lambda A B$ space (or $\lambda A K$ space), then $\sup _{n}\left\|x^{[n]_{\lambda}}\right\|_{X}<\infty$ (or $\lim _{n}\left\|x^{[n]_{\lambda}}-x\right\|_{X}=0$ ). Further, since the boundedness and weak boundedness are equal in normed spaces, one can easily see that $\sup _{n}\left|f\left(x^{[n]_{\lambda}}\right)\right|<\infty$ holds, for every $f \in X^{\prime}, x \in \Lambda B_{X}$.

For all $x \in \omega$, since $\left\{x^{[n]} \mid n \in \mathbf{N}\right\} \supset\left\{x_{\lambda}^{[n]} \mid n \in \mathbf{N}\right\}$, we have

$$
\Lambda \mathcal{P}_{X} \supset \mathcal{P}_{X}
$$

for the properties $\mathcal{P}=B, F, W, S$ and $\Lambda \mathcal{P}=\Lambda B, \Lambda F, \Lambda W, \Lambda S$.

In the other hand, let us define the operator $\Lambda=\left(\lambda_{n k}\right)$ associated with the sum operator

$$
s_{n k}=\left\{\begin{array}{lc}
1 & , \quad(1 \leq k \leq n) \\
0 & , \quad(k>n)
\end{array},\right.
$$

then we obtain the spaces

$$
\begin{aligned}
\lambda(B) & =\left\{x=\left(x_{j}\right) \in \omega: \sum_{j=1}^{k} x_{j} \in \ell_{\infty}^{\lambda}\right\} \\
& =\left\{x=\left(x_{j}\right) \in \omega: \sup _{n} \frac{1}{\lambda_{n}}\left|\sum_{k=1}^{n}\left(\lambda_{k}-\lambda_{k-1}\right) \sum_{j=1}^{k} x_{j}\right|<\infty\right\}
\end{aligned}
$$

and

$$
\begin{aligned}
\lambda(S) & =\left\{x=\left(x_{j}\right) \in \omega: \sum_{j=1}^{k} x_{j} \in c^{\lambda}\right\} \\
& =\left\{x=\left(x_{j}\right) \in \omega: \lim _{n} \frac{1}{\lambda_{n}}\left(\sum_{k=1}^{n}\left(\lambda_{k}-\lambda_{k-1}\right) \sum_{j=1}^{k} x_{j}\right) \text { exists }\right\}
\end{aligned}
$$


with the norm

$$
\|x\|_{\lambda(B)}=\|x\|_{\lambda(S)}=\sup _{n} \frac{1}{\lambda_{n}}\left|\sum_{k=1}^{n}\left(\lambda_{k}-\lambda_{k-1}\right) \sum_{j=1}^{k} x_{j}\right| .
$$

We define the $\lambda(B)$ and $\lambda(S)$ duals of a sequence space $X$ as

$$
\begin{aligned}
X^{\lambda(B)} & =\left\{x=\left(x_{j}\right) \in \omega: \sup _{n} \frac{1}{\lambda_{n}}\left|\sum_{k=1}^{n}\left(\Delta \lambda_{k}\right) \sum_{j=1}^{k} x_{j} y_{j}\right|<\infty, \forall y=\left(y_{j}\right) \in X\right\} \\
& =\left\{x=\left(x_{j}\right) \in w: x y \in \lambda(B), \forall y=\left(y_{j}\right) \in X\right\}
\end{aligned}
$$

and

$$
\begin{aligned}
X^{\lambda(S)} & =\left\{x=\left(x_{j}\right) \in \omega: \lim _{n} \frac{1}{\lambda_{n}}\left(\sum_{k=1}^{n}\left(\Delta \lambda_{k}\right) \sum_{j=1}^{k} x_{j} y_{j}\right) \text { exists, } \forall y=\left(y_{j}\right) \in X\right\} \\
& =\left\{x=\left(x_{j}\right) \in \omega: x y \in \lambda(S), \forall y=\left(y_{j}\right) \in X\right\}
\end{aligned}
$$

respectively. We have $X^{\lambda(S)} \subset X^{\lambda(B)}$ and if $\varsigma=\lambda(S), \lambda(B)$, then the inclusion $X \subset Y$ yields $Y^{\varsigma} \subset X^{\varsigma}$. We also have $X^{\varsigma}=X^{\varsigma \varsigma \varsigma}$ and $X \subset X^{\varsigma \varsigma}$. If $X=X^{\varsigma \varsigma}$, then $X$ is said to be a $\varsigma-$ space. In the sake of shortness, we use the notation $X^{\lambda(S) \lambda(S)}=X^{\lambda^{2}(S)}$ and $X^{\lambda(B) \lambda(B)}=X^{\lambda^{2}(B)}$. It can be easily seen that, if $\lambda_{k}=k$, one can obtain the spaces $\sigma s$ and $\sigma b$ from the spaces $\lambda(S)$ and $\lambda(B)$, respectively [4].

Proposition 2.1. The inclusions $c s \subset \lambda(S)$ and $b s \subset \lambda(B)$ hold. $\lambda(B) \subset \sigma b$ and $\lambda(S) \subset \sigma s$ if and only if the condition (1.2) holds.

Proof. It is clear.

Theorem 2.2. If $X$ is an $A K$-space, then it is an $\lambda A K$-space.

Proof. It is clear with Stolz-Cesàro theorem.

Let $X \supset \phi$ be a $B K$-space. If the following conditions hold then it is said to be $X$ has a monotone norm [19]:

i. For $n<m\left\|x^{[n]}\right\| \leq\left\|x^{[m]}\right\|$,

ii. $\|x\|=\sup _{m}\left\|x^{[m]}\right\|$.

Theorem 2.3. $c^{\lambda}$ has monoton norm.

Proof. Since $\Lambda$ is a triangle $c^{\lambda}$ is a $B K$-space. Let $x$ be fixed and $\Lambda(m, n)=$ $\left|\frac{1}{\lambda_{n}} \sum_{k=1}^{m}\left(\Delta \lambda_{k}\right) x_{k}\right|$. So, from $\|x\|_{\lambda^{\infty}}=\sup _{n}\left|\frac{1}{\lambda_{n}} \sum_{k=1}^{n}\left(\Delta \lambda_{k}\right) x_{k}\right|$, we have $\|x\|_{\lambda^{\infty}}=$ $\lambda(n, n)$. Since, for $x^{[m]}=\left\{x_{1}, \ldots, x_{m}, 0,0, \ldots\right\}$

$$
\left|\Lambda\left(x^{[m]}\right)_{n}\right|=\left\{\begin{array}{cc}
\Lambda(n, n) & , \quad n \leq m \\
\Lambda(m, n) & , \quad n \geq m
\end{array},\right.
$$

we also have $\Lambda(m, n)$ is decreasing for $n$. Therefore, the first condition holds. In the other hand, from $\left\|x^{[m]}\right\|_{\lambda^{\infty}}=\sup _{n}\{\Lambda(n, n): n \leq m\}$, one can easily see that, the second condition also holds.

Theorem 2.4. Let $X \supset \phi$ be an FK-space. Then, the inclusions

$$
\phi \subset \Lambda S_{X} \subseteq \Lambda W_{X} \subset \Lambda F_{X} \subset \Lambda B_{X} \subset X
$$


and

$$
\phi \subset \Lambda S_{X} \subseteq \Lambda W_{X} \subset \bar{\phi}
$$

hold.

Proof. From the definitions of the spaces $\phi, \Lambda S_{X}, \Lambda W_{X}, \Lambda F_{X}, \Lambda B_{X}$, we have $x_{\lambda}^{[n]} \rightarrow x,($ by the norm of $X) \Rightarrow f\left(x_{\lambda}^{[n]}\right) \rightarrow f(x) \Rightarrow\left(f\left(x_{\lambda}^{[n]}\right)\right) \in c \Rightarrow\left(f\left(x_{\lambda}^{[n]}\right)\right) \in \ell_{\infty}$, for every $f \in X^{\prime}$.

Now, we shall prove that $\Lambda W_{X} \subset \bar{\phi}$. Let us suppose that $x \in \Lambda W_{X}$. So,

$$
f(x)=\lim _{n} \frac{1}{\lambda_{n}} \sum_{k=1}^{n} \Delta \lambda_{k} f\left(x^{[k]}\right),
$$

holds for every $f \in X^{\prime}$. Therefore, we have the result from Hahn - Banach theorem[19].

Theorem 2.5. Distinguished subspaces of an FK-space are monotone. That is,

$$
X \subset Y \Rightarrow \Omega_{X} \subset \Omega_{Y}
$$

holds for every $\Omega=\Lambda S, \Lambda W, \Lambda F, \Lambda B$.

Proof. Since the others are similar, we only give the proof for $\lambda A K$ property. By bearing in mind that the inclusion map is continuous, let us suppose that $X \subset Y$ and $x \in \Lambda S_{X}$. Therefore, the convergence $\frac{1}{\lambda_{n}} \sum_{k=1}^{n}\left(\Delta \lambda_{k}\right) x^{[k]} \rightarrow x$, in $X$ yields that the convergence $\frac{1}{\lambda_{n}} \sum_{k=1}^{n}\left(\Delta \lambda_{k}\right) x^{[k]} \rightarrow x$, in $Y$. This completes the proof.

Theorem 2.6. Let each $X_{i} \supset \phi,(i=1,2, \ldots, m)$ be $F K$ spaces with paranorms $p^{(i)},(i=1,2, \ldots, m)$ and $X=\sum_{i=1}^{m} X_{i}$. If $\Omega=\Lambda S, \Lambda W, \Lambda F, \Lambda B$, then $\sum_{i=1}^{m} \Omega_{X_{i}} \subseteq \Omega_{X}$ holds.

Proof. Since the others are similar, we only give proof for $\lambda A K$ property. Let us suppose that $x^{(i)} \in \Lambda S_{X_{i}}(i=1,2, \ldots, m)$. Then,

$$
p^{(1)}\left[\left(x^{(1)}\right)_{\lambda}^{[n]}-x^{(1)}\right] \rightarrow 0, \ldots, p^{(m)}\left[\left(x^{(m)}\right)_{\lambda}^{[n]}-x^{(m)}\right] \rightarrow 0,
$$

that is, by taking $\left\{p^{(i)}\left[\left(x^{(i)}\right)_{\lambda}^{[n]}-x^{(i)}\right] \rightarrow 0\right\}_{i=1}^{m}$, we have

$$
\begin{aligned}
q\left[\left(\sum_{i=1}^{m} x^{(i)}\right)_{\lambda}^{[n]}-\left(\sum_{i=1}^{m} x^{(i)}\right)\right] & =q\left[\sum_{i=1}^{m}\left(\left(x^{(i)}\right)_{\lambda}^{[n]}-x^{(i)}\right)\right] \\
& =q\left\{\sum_{i=1}^{m}\left(\frac{1}{\lambda_{n}} \sum_{k=1}^{n}\left(\Delta \lambda_{k}\right)\left(x^{(i)}\right)^{[k]}\right)-\left(x^{(i)}\right)\right\} \\
& \leq p^{(1)}\left\{\left(\frac{1}{\lambda_{n}} \sum_{k=1}^{n}\left(\Delta \lambda_{k}\right)\left(x^{(1)}\right)^{[k]}\right)-\left(x^{(1)}\right)\right\}+ \\
& +\ldots+ \\
& +p^{(m)}\left\{\left(\frac{1}{\lambda_{n}} \sum_{k=1}^{n}\left(\Delta \lambda_{k}\right)\left(x^{(m)}\right)^{[k]}\right)-\left(x^{(m)}\right)\right\} \\
& =p^{(1)}\left[\left(x^{(1)}\right)_{\lambda}^{[n]}-x^{(1)}\right]+\ldots+p^{(m)}\left[\left(x^{(m)}\right)_{\lambda}^{[n]}-x^{(m)}\right] \\
& \rightarrow 0, \text { as } n \rightarrow \infty .
\end{aligned}
$$

Therefore, we have $\sum_{i=1}^{m} x^{(i)} \in \Lambda S_{X}$. This completes the proof (see also [8]). 
Theorem 2.7. Let $\left\{X_{n}\right\}_{n=1}^{\infty}$ be a sequence of $F K$-spaces and $X=\bigcap_{n} X_{n}$. Then we have, $\Omega_{X}=\bigcap_{n} \Omega_{X_{n}}$ for $\Omega=\Lambda S, \Lambda W, \Lambda F, \Lambda B$.

Proof. By monotonicity, $\Omega_{X} \subseteq \Omega_{X_{n}}$, and so for $\Omega=\Lambda S, \Lambda W, \Lambda F, \Lambda B$ we have $\Omega_{X} \subseteq \bigcap_{n} \Omega_{X_{n}}$, for all $n \in \mathbb{N}$. Since the others are similar, we shall only prove that $\bigcap_{n} \Omega_{X_{n}} \subseteq \Omega_{X}$ holds for $\Omega=\Lambda S$. Let us suppose that $x \in \bigcap_{n} \Lambda S_{X_{n}}$. Then for all $n, k \in \mathbf{N}, q_{n k}\left(x_{\lambda}^{[n]}-x\right) \rightarrow 0$ and also $x_{\lambda}^{[n]} \rightarrow x$ in $X$, we have $x \in \Lambda S_{X}$. This completes the proof (see also [8]).

\section{DuAls}

In the following, we give some relationship between the distinguished subspaces and $f-, \lambda(S)$ ve $\lambda(B)$ duals for an $F K$-space $X \supset \phi$.

Theorem 3.1. Let $X \supset \phi$ be an FK-space. Then we have

$$
\Lambda B_{X}^{+}=X^{f \lambda(B)} \text { and } \Lambda F_{X}^{+}=X^{f \lambda(S)} .
$$

Proof. We know that $z \in \Lambda B_{X}^{+}$if and only if $\left(z_{n} f\left(\delta^{n}\right)\right)_{n \in \mathbf{N}} \in \lambda(B)$, for all $f \in X^{\prime}$. Let us take $\left(f\left(\delta^{n}\right)\right)_{n \in \mathbf{N}} \in X^{f}$, for some $f \in X^{\prime}$, then from the definition of $\lambda(B)$, we have $z \in X^{f \lambda(B)}$. The other one is similar.

Corollary 3.2. Let $X \supset \phi$ be an $F K$-space. Then, the spaces $\Lambda B_{X}^{+}$and $\Lambda F_{X}^{+}$are $\lambda(B)$ and $\lambda(S)$ spaces, respectively.

Theorem 3.3. Let $X \supset \phi$ be an FK-space and $\bar{\phi}$ is the closure of $\phi$ in $X$. If $\bar{\phi} \subset Y \subset X$, then

$$
\Lambda B_{X}^{+}=\Lambda B_{Y}^{+} \quad \text { and } \Lambda F_{X}^{+}=\Lambda F_{Y}^{+} .
$$

Proof. Since $\bar{\phi} \subset Y \subset X$ holds, we have $\Lambda B_{\bar{\phi}}^{+} \subset \Lambda B_{Y}^{+} \subset \Lambda B_{X}^{+}$. Therefore, for an arbitrary $F K$-space $X \supset \phi$, we have $(\bar{\phi})^{f}=X^{f}$, and so $\bar{\phi}^{f} \subset Y^{f} \subset X^{f}=\bar{\phi}^{f}$. Anymore, we get desired result by taking $\lambda(B)$ dual in both sides. Similarly, we can prove that $\Lambda F_{X}^{+}=\Lambda F_{Y}^{+}$.

Theorem 3.4. Let $X \supset \phi$ be an FK-space. Then,

$$
X \text { is an } \Lambda B \text { space } \Leftrightarrow X^{f} \subset X^{\lambda(B)}
$$

and

$$
X \text { is an } \Lambda F \text { space } \Leftrightarrow X^{f} \subset X^{\lambda(S)} .
$$

Proof. $\{\Rightarrow\}$ : By hypothesis and previous result, for $\lambda(B)$ and $\lambda(S)$ duals of an $F K$-space, we have $X \subset \Lambda B_{X}^{+}=X^{f \lambda(B)}$ and $X \subset \Lambda F_{X}^{+}=X^{f \lambda(S)}$. From taking $\lambda(B)$ and $\lambda(S)$ duals,

$$
X^{f \lambda^{2}(B)} \subset X^{\lambda(B)}
$$

and

$$
X^{f \lambda^{2}(S)} \subset X^{\lambda(S)}
$$

hold. Now, we have also $X^{f} \subset X^{f \lambda^{2}(B)}$ and $X^{f} \subset X^{f \lambda^{2}(S)}$, then

$$
X^{f} \subset X^{\lambda(B)}
$$

and

$$
X^{f} \subset X^{\lambda(S)}
$$

hold. 
$\{\Leftarrow\}$ : In hypothesis, by using the inclusions $X \supset \phi X^{f} \subset X^{\lambda(B)}$ and $X^{f} \subset$ $X^{\lambda(S)}$, let us take $\lambda(B)$ and $\lambda(S)$ duals. From the properties of $\lambda(B)$ and $\lambda(S)$ duals, we have

$$
X \subset X^{\lambda^{2}(S)} \subset X^{f \lambda(B)}=\Lambda B_{X}^{+}
$$

and

$$
X \subset X^{\lambda^{2}(S)} \subset X^{f \lambda(S)}=\Lambda F_{X}^{+},
$$

respectively. This means that, $X$ is a $\lambda A B$ and $F \lambda A K$ space, respectively.

As a result of this theorem, we have following since $X^{\lambda(S)}$ is a closed subspace of $X^{\lambda(B)}$ space (see also [19]).

Corollary 3.5. Let $X \supset \phi$ be a $B K-\lambda A B$-space, then $X^{\lambda(S)}$ is closed in $\lambda^{f}$.

Theorem 3.6. The following assertions for an $F K$-space $X \supset \phi$ are true:

(i) If $X$ is an F $\lambda A K$-space, then $X^{f}=X^{\lambda(S)}$,

(ii) If $X$ is an $A D$-space, then $X^{\lambda(S)}=X^{\lambda(B)}$,

(iii) The inclusions $X^{\beta} \subset X^{\lambda(S)} \subset X^{\lambda(B)} \subset X^{f}$ are hold.

Proof. (i) Let us suppose that $y \in X^{\lambda(S)}$ and

$$
f(x)=\lim _{n} \frac{1}{\lambda_{n}} \sum_{k=1}^{n} \Delta \lambda_{k} \sum_{j=1}^{k} x_{j} y_{j}
$$

holds, for every $x \in X$. By Banach-Steinhaus theorem, we have $f \in X^{\prime}$. Since we have

$$
\begin{aligned}
f(x) & =\lim _{n} \frac{1}{\lambda_{n}} \sum_{k=1}^{n} \Delta \lambda_{k} \sum_{j=1}^{k} x_{j} y_{j} \\
& =\lim _{n} \frac{1}{\lambda_{n}}\left(\lambda_{n} \sum_{k=1}^{n} x_{k} y_{k}-\sum_{k=1}^{n} \lambda_{k-1} x_{k} y_{k}\right) \\
& =\lim _{n}\left(\sum_{k=1}^{n} x_{k} y_{k}-\frac{1}{\lambda_{n}} \sum_{k=1}^{n} \lambda_{k-1} x_{k} y_{k}\right),
\end{aligned}
$$

by taking $x=\delta^{m}$, we have

$$
\begin{aligned}
f\left(\delta^{m}\right) & =\lim _{n}\left(y_{m}-\frac{\lambda_{m-1}}{\lambda_{n}} y_{m}\right) \\
& =\lim _{n}\left(y_{m}\left(1-\frac{\lambda_{m-1}}{\lambda_{n}}\right)\right) \\
& =y_{m}, m<n .
\end{aligned}
$$

and then $y=\left(y_{m}\right) \in X^{f}$. This means that, $X^{\lambda(S)} \subseteq X^{f}$.

In the other hand, let us take $y \in X^{f}$. Since $X$ is an $F \lambda A K$-space,

$$
\lim _{n} \frac{1}{\lambda_{n}} \sum_{k=1}^{n} \Delta \lambda_{k} f\left(x^{[k]}\right)
$$


exists, and so $y=\left(y_{j}\right)=\left(f\left(\delta^{j}\right)\right) \in X^{\lambda(S)}$, for all $x \in X$. Therefore, $X^{f} \subseteq X^{\lambda(S)}$.

(ii) It is enough to show that, if $X$ is an $A D$-space, then $X^{\lambda(B)} \subset X^{\lambda(S)}$ holds.

Let us suppose that $y \in X^{\lambda(B)}$ and define $\left\{f_{n}\right\}$ as,

$$
f_{n}(x)=\lim _{n} \frac{1}{\lambda_{n}} \sum_{k=1}^{n} \Delta \lambda_{k} \sum_{j=1}^{k} x_{j} y_{j},
$$

for all $x \in X$. Then $\left\{f_{n}\right\}$ is point-wise bounded and so is equicontinuous [19].

For all $m \leq n$,

$$
\lim _{n} f_{n}\left(\delta^{m}\right)=y_{m}
$$

and so is $\phi \subset\left\{x: \lim _{n} f_{n}(x)\right.$ mevcut $\}$. By convergence lemma [19], $\left\{x: \lim _{n} f_{n}(x)\right.$ mevcut $\}$ is a closed subspace of $X$. Since $X$ is an $A D$-space, we have

$$
\phi \subset\left\{x: \lim _{n} f_{n}(x) \text { exists }\right\}=\bar{\phi}=X .
$$

That is, $y \in X^{\lambda(S)}$. Hence, $X^{\lambda(S)}=X^{\lambda(B)}$.

(iii) It is enough that, the inclusion $X^{\lambda(B)} \subset \lambda^{f}$ holds. For $\bar{\phi} \subset X$,

$$
\begin{aligned}
X^{\lambda(B)} & \subset(\bar{\phi})^{\lambda(B)} \\
& =(\bar{\phi})^{\lambda(S)} \\
& \subset(\bar{\phi})^{f} \\
& =X^{f},
\end{aligned}
$$

since $\bar{\phi}$ is an $A D$-space.

We have the following corollary by using previous theorems.

Corollary 3.7. Let $X \supset \phi$ be an FK-space. Then,

$$
X \text { is a } \lambda A B \text { space } \Leftrightarrow X^{f}=X^{\lambda(B)}
$$

and

$$
X \text { is a } F \lambda A K \text { space } \Leftrightarrow X^{f}=X^{\lambda(S)} .
$$

Theorem 3.8. Let $X \supset \phi$ be an $F K-\lambda A B$-space. Then, $\bar{\phi}$ is a $\lambda A K$-space and the equality

$$
\Lambda S_{X}=\Lambda W_{X}=\bar{\phi}
$$

holds.

Proof. Since we get the proof by the similar way used in the proof of given theorem in [7], we omit the details.

Theorem 3.9. Let $X \supset \phi$ be an FK-space such that $\bar{\phi}$ is a $\lambda A K$-space. Then,

$$
\Lambda F_{X}^{+}=\bar{\phi}^{\lambda^{2}(S)} .
$$

Proof. We know that $\Lambda F_{X}^{+}=X^{f \lambda(S)}$ and $X^{f}=(\bar{\phi})^{f}$ for an $F K$-space $X \supset \phi$. Now, by taking $\lambda(S)$ dual in both sides, we have $X^{f \lambda(S)}=(\bar{\phi})^{f \lambda(S)}$.

In this theorem, we can replace $\bar{\phi}, \mathrm{s} \lambda A K$ property with the weaker property $F \lambda A K$. Because, if $X \supset \phi$ is an $F \lambda A K$-space, then $X^{f}=X^{\lambda(S)}$. 
Corollary 3.10. Let $X \supset \phi$ be an FK-space. Then, $X$ is an $F \lambda A K$ space $\Leftrightarrow \bar{\phi}$ is a $\lambda A K$ space and $X \subset \bar{\phi}^{\lambda^{2}(S)}$.

Theorem 3.11. Let $X \supset \phi$ be an FK-space. Then the following are equivalent:

$$
\begin{aligned}
\text { (i) } & X \text { is an } F \lambda A K \text { space, } \\
\text { (ii) } & X \subset \Lambda F_{X}^{\lambda^{2}(S)}, \\
\text { (iii) } & X \subset \Lambda W_{X}^{\lambda^{2}(S)}, \\
\text { (iv) } & X \subset \Lambda S_{X}^{\lambda^{2}(S)}, \\
\text { (v) } & X^{\lambda(S)}=\Lambda F_{X}^{\lambda(S)}=\Lambda W_{X}^{\lambda(S)}=\Lambda S_{X}^{\lambda(S)} .
\end{aligned}
$$

Proof. $(i v) \Rightarrow(i i i) \Rightarrow(i i)$ are clear from the definitions of these spaces.

$(i i) \Rightarrow(i)$ : Let us suppose that $X \subset \Lambda F_{X}^{\lambda^{2}(S)}$. Then,

$$
X^{f} \subset X^{f \lambda^{2}(S)}=\Lambda F_{X}^{+\lambda(S)} \subset \Lambda F_{X}^{\lambda(S)} \subset X^{\lambda(S)}
$$

hold.

$(i) \Rightarrow(i v)$ : It is clear from previous results.

$(i v) \Rightarrow(v)$ : We have for an $F K$-space $X \supset \phi$;

$$
\Lambda S_{X} \subset \Lambda W_{X} \subset \Lambda F_{X} \subset X .
$$

By taking $\lambda(S)$ dual in every side, we have

$$
X^{\lambda(S)} \subset \Lambda F_{X}^{\lambda(S)} \subset \Lambda W_{X}^{\lambda(S)} \subset \Lambda S_{X}^{\lambda(S)} .
$$

By bearing in mind the hypothesis with the previous results, we get the proof.

$(v) \Rightarrow(i v)$ : It is clear.

Let $X$ be an $F K$-space has $\lambda A K$ property. The following theorem tells us that there is a closed relationship between the spaces $X^{\lambda(S)}$ and $X^{\prime}$.

Theorem 3.12. Let $X \supset \phi$ be an FK-space. Then the following are equivalent:

(i) $X$ is an $S \lambda A K$,

(ii) $X$ is a $\lambda A K$,

(iii) $\quad X^{\lambda(S)} \widehat{=} X^{\prime},\left(f \rightarrow f\left(\delta^{k}\right)\right)$.

Proof. $(i) \Rightarrow($ ii): If $X$ is an $S \lambda A K$ space, then it is an $A D$-space and also is a $\lambda A B$-space. Therefore, $X$ is a $\lambda A K$-space.

$(i i) \Rightarrow(i i i)$ : Since $X$ is a $\lambda A K$-space, then it is $A D$-space, and so $X^{f}=X^{\prime}$ holds.

(iii) $\Rightarrow(i)$ : Let us suppose that $u \in X^{\lambda(S)}$. Then, for all $f \in X^{\prime}$ and $x \in X$, we have

$$
f(x)=\lim _{n} \frac{1}{\lambda_{n}} \sum_{k=1}^{n}\left(\Delta \lambda_{k}\right) \sum_{j=1}^{k} u_{j} x_{j} .
$$

Therefore, we have $x \in \Lambda W_{X}$. 
Theorem 3.13. Let $X \supset \phi$ be an FK-space. Then, the following assertions are equivalent:

$$
\begin{aligned}
\text { (i) } & \Lambda W_{X} \text { is closed in } X, \\
\text { (ii) } & \bar{\phi} \subset \Lambda B_{X}, \\
\text { (iii) } & \bar{\phi} \subset \Lambda F_{X}, \\
\text { (iv) } & \bar{\phi}=\Lambda W_{X}, \\
\text { (v) } & \bar{\phi}=\Lambda S_{X} \\
\text { (vi) } & \Lambda S_{X} \text { is closed in } X .
\end{aligned}
$$

Proof. $(v) \Rightarrow(i v),(i v) \Rightarrow(i i i),(v) \Rightarrow(i i)$ and $(i i i) \Rightarrow(i i)$ are clear. Since $\bar{\phi}$ is a $\lambda A K$-space, we have $\bar{\phi} \subset \Lambda S_{X}$, and so $(i i) \Rightarrow(v)$ holds.

In the other hand, from $\phi \subset \Lambda S_{X} \subset \Lambda W_{X} \subset \bar{\phi}$, we have $(i) \Rightarrow$ (iv) and $(v i) \Rightarrow(v) .(i v) \Rightarrow(i)$ and $(v) \Rightarrow(v i)$ are also clear from the previous theorems and corollaries.

\section{REFERENCES}

[1] B. Altay and F. Başar, Certain topological properties and duals of the matrix domain of a triangle matrix in a sequence space, J. Math. Anal. Appl. 336(1)(2007), 632-645.

[2] F. Başar, Summability Theory and Its Applications, Bentham Science Publishers, İstanbul (2012).

[3] J. Boos, Classical and Modern Methods in Summability, Oxford University Press. New York, Oxford, 2000.

[4] M. Buntinas, Convergent and bounded Cesàro sections in FK-spaces, Math. Z., 121 (1971), 191-200.

[5] M. Buntinas, On sectionally dense summability fields, Math. Zeitschr., 132 (1973), 141-149.

[6] M. Buntinas, On Toeplitz sections in sequence spaces, Math. Proc. Cambridge Philos. Soc., 78 (1975), 451-460.

[7] İ. Dağadur, On some subspaces of an FK-space, Math. Commun., Vol.7, (2002), 15-20.

[8] R. Devos, Combinations of distinguished subsets and conullity, Math. Z., 192 (1986), 447-451.

[9] D. J. Fleming and J. C. Magee, FK-multiplier spaces, Proc. Amer. Math. Soc., 125(1)(1997), 175-181.

[10] D. J. H. Garling, The $\beta$ - and $\gamma$-duality of sequence spaces, Math. Proc. Cambridge Philos. Soc., 63 (Jan. 1967), 963-981.

[11] D. J. H. Garling, On topological sequence spaces, Math. Proc. Cambridge Philos. Soc., 63 (1967), 997-1019.

[12] G. Goes and S. Goes, Sequences of bounded variation and sequences of fourier coefficients. I, Math. Z.,118(1970), 93-102.

[13] G. Goes, Summen von FK-räumen funktionale abschnittskonvergenz und umkehrsatz, Tohoku. Math. J., 26(1974), 487-504.

[14] K-G., Grosse-Erdmann, On $l^{1}$-Invariant Sequence Spaces, J. Math. Anal. Appl., 262(2001), 112-132.

[15] M. Mursaleen A. K. Noman, On the spaces of $\lambda$-convergent and bounded sequences, Thai J. Math. 8 (2) (2010), 311-329.

[16] E. Malkowsky, Recent results in the theory of matrix transformations in sequence spaces, Mat. Vesnik 49(1997), 187-196.

[17] A. Wilansky, Functional Analysis, Blaisdell Press, 1964.

[18] A. Wilansky, Modern Methods in Topological Vector Spaces, McGraw Hill, New York, 1978.

[19] A. Wilansky, Summability Through Functional Analysis, North-Holland, Amsterdam, 1984.

[20] K. Zeller, Allgemeine eigenschaften von limitierungsverfahren, Math. Z., 53 (1951), 463-487. 
Van Yüzüncü Yil University, Faculty of Science, Department of Mathematics, 65080, VAN, TURKEY

Van Yüzüncü Yil University, Faculty of Education, Department of Mathematics Education, 65080, Van, Turkey 